\title{
On limiting embeddings of Besov spaces
}

\author{
by \\ V. I. Kolyada (Karlstad) and A. K. Lerner (Ramat Gan)
}

\begin{abstract}
We investigate the classical embedding $B_{p, \theta}^{s} \subset B_{q, \theta}^{s-n(1 / p-1 / q)}$. The sharp asymptotic behaviour as $s \rightarrow 1$ of the operator norm of this embedding is found. In particular, our result yields a refinement of the Bourgain, Brezis and Mironescu theorem concerning an analogous problem for the Sobolev-type embedding. We also give a different, elementary proof of the latter theorem.
\end{abstract}

1. Introduction. For $0<s<1$ and $1 \leq p, \theta<\infty$ the Besov space $B_{p, \theta}^{s}\left(\mathbb{R}^{n}\right)$ consists of all functions $f \in L^{p}\left(\mathbb{R}^{n}\right)$ such that

$$
\|f\|_{b_{p, \theta}^{s}} \equiv\left(\int_{0}^{\infty}\left(t^{-s} \omega_{p}(f, t)\right)^{\theta} \frac{d t}{t}\right)^{1 / \theta}<\infty,
$$

where $\omega_{p}(f, t)$ is the $L^{p}$-modulus of continuity of $f$. Set also $B_{p}^{s} \equiv B_{p, p}^{s}$.

One of the basic inequalities of embedding theory, which goes back to the classical Hardy-Littlewood theorem on Lipschitz classes, says that if $\gamma \equiv n(1 / p-1 / q)<s$, then $($ see $[11, \S 6.3])$

$$
\|f\|_{b_{q, \theta}^{s-\gamma}} \leq c\|f\|_{b_{p, \theta}^{s}} \quad(1 \leq p<q<\infty, 1 \leq \theta<\infty) .
$$

Note that a simple argument shows that $\|f\|_{b_{p, \theta}^{s}} \rightarrow \infty$ as $s \rightarrow 1$, whenever $f \nsim 0$. The aim of this paper is to study the asymptotic behaviour of the optimal constant $c$ in (1) as $s \rightarrow 1-0$. Our main result is the following.

Theorem 1.1. Let $0<s<1$ and $p<q<\infty$. Let also $\gamma \equiv n(1 / p-1 / q)$. Assume that $\gamma<s$ and $1 \leq \theta<\infty$. If either $p>1, n \geq 1$ or $p \geq 1, n \geq 2$, then for any $f \in B_{p, \theta}^{s}\left(\mathbb{R}^{n}\right)$ we have

$$
\|f\|_{b_{q, \theta}^{s-\gamma}} \leq A \frac{(1-s)^{1 / \theta^{*}}}{(s-\gamma)^{1 / \theta}}\|f\|_{b_{p, \theta}^{s},},
$$

where $\theta^{*}=\max (p, \theta)$ and the constant $A$ does not depend on $s$ and $f$.

2000 Mathematics Subject Classification: 46E30, $46 \mathrm{E} 35$.

Key words and phrases: Besov space, embedding theorem, rearrangement estimate, modulus of continuity. 
This theorem fails for $p=n=1$. We will also show that the exponent $1 / \theta^{*}$ is sharp in a sense.

We should mention that this work was inspired by recent results of Bourgain, Brezis, and Mironescu [1,2] and Maz'ya and Shaposhnikova [10]. Let $W_{p}^{1}\left(\mathbb{R}^{n}\right)$ be the Sobolev space of all $f \in L^{p}\left(\mathbb{R}^{n}\right)$ for which every first-order weak derivative exists and belongs to $L^{p}\left(\mathbb{R}^{n}\right)$. First, it was observed in [1] that there exists a limiting relation between Sobolev and Besov norms, that is, for any $f \in W_{p}^{1}\left(\mathbb{R}^{n}\right)$,

$$
\lim _{s \rightarrow 1-0}(1-s)\|f\|_{b_{p}^{s}}^{p} \asymp\|\nabla f\|_{L^{p}}^{p} .
$$

The main result of [2] concerns the well known Sobolev-type embedding $B_{p}^{s} \subset L^{q^{*}}, q^{*}=n p /(n-s p)$, proved in the late sixties independently by several authors (for the references, see [3, §18], [7, Sect. 10], [8, p. 56]). In [2], the sharp asymptotics of the best constant as $s \rightarrow 1$ in a related inequality was found, namely, if $1 / 2 \leq s<1$ and $1 \leq p<n / s$, then for any $f \in B_{p}^{s}\left(\mathbb{R}^{n}\right)$,

$$
\|f\|_{L^{q^{*}}}^{p} \leq c_{n} \frac{1-s}{(n-s p)^{p-1}}\|f\|_{b_{p}^{s}}^{p} \quad\left(q^{*}=\frac{n p}{n-s p}\right),
$$

where the constant $c_{n}$ depends only on $n$. In view of (3), the classical Sobolev inequality

$$
\|f\|_{L^{n p /(n-p)}} \leq c\|\nabla f\|_{p}, \quad 1 \leq p<n,
$$

can be considered as a limiting case of (4).

Similarly, Theorem 1.1 allows us to find a relation between (1) and the following result: if either $p>1, n \geq 1$ or $p \geq 1, n \geq 2$, and $\gamma \equiv n(1 / p-$ $1 / q)<1$, then for any $f \in W_{p}^{1}\left(\mathbb{R}^{n}\right)$,

$$
\|f\|_{b_{q, p}^{1-\gamma}} \leq c\|\nabla f\|_{p} \quad(p<q<\infty) .
$$

For $p>1$ this estimate was proved by Il'in [3, Theorem 18.12]. In the case $p \geq 1, n \geq 2$ the proof was given in [6]. By (3), Theorem 1.1 shows that inequality (5) is a limiting case of (2).

We now return to inequality (4). Note that the proof given in [2] is quite complicated. Afterwards, Maz'ya and Shaposhnikova [10] gave a simpler proof of this result. Also they studied the behaviour of the optimal constant as $s \rightarrow 0$. More precisely, they proved that the constant in (4) can be replaced by $c_{p, n} s(1-s)(n-s p)^{1-p}$. An important point of the method in $[10]$ is the fact that the spherically symmetric rearrangement is bounded in Besov spaces. Observe that the proof of this fact (due to Wik [15]) is rather difficult. However, it was shown in $[5,14]$ that sharp theorems on embedding into Orlicz classes can be directly obtained from estimates of rearrangements via moduli of continuity. The proof of these estimates is much simpler than that of Wik's theorem mentioned above. 
In this paper we show that (4) as well as a more general result of [10] can be immediately derived from the rearrangement estimates obtained in [5]. Moreover, we show that a stronger inequality with the Lorentz $L^{q^{*}, p}$-norm on the left-hand side of (4) holds: for any $0<s<1$ and $1 \leq p<n / s$,

$$
\|f\|_{L^{q^{*}, p}\left(\mathbb{R}^{n}\right)}^{p} \leq c_{p, n} \frac{s(1-s)}{(n-s p)^{p}}\|f\|_{b_{p}^{s}\left(\mathbb{R}^{n}\right)}^{p} .
$$

It is easy to see, by the standard relation between Lebesgue and Lorentz norms, that (6) implies (4). Note that (6) is contained implicitly in [10].

Observe that estimates of Lorentz norms via Besov norms (without the sharp asymptotics of the best constant) are also well known $[4,12]$ (see Section 2 below). We show that to get (6) for $1 / 2<s<1$, it suffices to use these estimates with some intermediate Besov norm and then apply Theorem 1.1. Thus, this theorem is a more precise result than (6) in the cases $p \neq 1$ or $n \neq 1,1 / 2<s<1$.

We also note that the papers $[1,2]$ deal with a slightly different definition of the $B_{p}^{s}$-norm. However it is well known that these definitions are equivalent with corresponding constants not depending on $s$. We recall briefly the proof of this fact below.

The paper is organized as follows. Section 2 contains necessary information and some auxiliary results concerning Hardy-type inequalities, moduli of continuity, and non-increasing rearrangements. In Section 3 we prove Theorem 1.1, and in Section 4 we prove (6).

\section{Auxiliary results}

2.1. Hardy-type inequalities. We will use the following lemma of Hardy [13, p. 196].

Lemma 2.1. Let $\alpha>0$ and $1 \leq p<\infty$. Then for any non-negative measurable function $\varphi$ on $(0, \infty)$,

$$
\left(\int_{0}^{\infty}\left(\int_{0}^{t} \varphi(u) d u\right)^{p} t^{-\alpha-1} d t\right)^{1 / p} \leq \frac{p}{\alpha}\left(\int_{0}^{\infty}(t \varphi(t))^{p} t^{-\alpha-1} d t\right)^{1 / p}
$$

and

$$
\left(\int_{0}^{\infty}\left(\int_{t}^{\infty} \varphi(u) d u\right)^{p} t^{\alpha-1} d t\right)^{1 / p} \leq \frac{p}{\alpha}\left(\int_{0}^{\infty}(t \varphi(t))^{p} t^{\alpha-1} d t\right)^{1 / p} .
$$

We say that a measurable function $f$ on $(0, \infty)$ is quasi-decreasing with constant $c>0$ if $f\left(t_{1}\right) \leq c f\left(t_{2}\right)$ whenever $0<t_{2}<t_{1}<\infty$.

We will need a Hardy-type inequality for quasi-decreasing functions in the case $0<p<1$. Though the following statement is apparently known, we shall prove it for the sake of completeness. 
Proposition 2.2. Let $f$ be a non-negative, quasi-decreasing function on $(0, \infty)$ with constant $c$. Suppose also that $\alpha>0, \beta>-1$ and $0<p<1$. Then

$$
\int_{0}^{\infty} u^{-\alpha-1}\left(\int_{0}^{u} f(t) t^{\beta} d t\right)^{p} d u \leq \frac{(c(1+\beta))^{1-p} p}{\alpha} \int_{0}^{\infty} u^{-\alpha-1}\left(f(u) u^{\beta+1}\right)^{p} d u
$$

Proof. We can assume that $(1+\beta) p>\alpha$, otherwise the integral on the right-hand side of (9) diverges. Also, one can suppose that $f$ is bounded and compactly supported. Define $F(u)=\int_{0}^{u} f(t) t^{\beta} d t$. Note that $F(u) \geq$ $f(u) u^{\beta+1} / c(\beta+1)$. Therefore, integrating by parts yields

$$
\begin{aligned}
\int_{0}^{\infty} u^{-\alpha-1}(F(u))^{p} d u & =\frac{p}{\alpha} \int_{0}^{\infty} u^{-\alpha+\beta} f(u)(F(u))^{p-1} d u \\
& \leq \frac{(c(1+\beta))^{1-p} p}{\alpha} \int_{0}^{\infty} u^{-\alpha-1}\left(f(u) u^{\beta+1}\right)^{p} d u
\end{aligned}
$$

as required.

2.2. Moduli of continuity. For any $f \in L^{p}\left(\mathbb{R}^{n}\right), 1 \leq p<\infty$, and $h \in \mathbb{R}^{n}$, set

$$
I_{p}(h)=\left(\int_{\mathbb{R}^{n}}|f(x+h)-f(x)|^{p} d x\right)^{1 / p} .
$$

The modulus of continuity of a function $f$ is defined by

$$
\omega_{p}(f, \delta)=\sup _{|h| \leq \delta} I_{p}(h) \quad(0<\delta<\infty) .
$$

Observe that $\omega_{p}(f, \delta)$ is a non-decreasing and subadditive function. In particular, this implies that $\omega_{p}(f, \delta) / \delta$ is quasi-decreasing on $(0, \infty)$ with constant 2 .

We wish to point out that the papers $[2,10]$ deal with the seminorm

$$
\|f\|_{w_{p}^{s}} \equiv\left(\int_{\mathbb{R}^{n}} \int_{\mathbb{R}^{n}} \frac{|f(x)-f(y)|^{p}}{|x-y|^{n+s p}} d x d y\right)^{1 / p}
$$

in place of $\|f\|_{b_{p}^{s}}$. It is well known that these seminorms are equivalent. We outline the proof of this fact just in order to show that the corresponding constants do not depend on $s$.

Proposition 2.3. For any $f \in B_{p}^{s}\left(\mathbb{R}^{n}\right)$ and all $s \in(0,1)$,

$$
\left(n v_{n}\right)^{1 / p} 2^{-n-2}\|f\|_{b_{p}^{s}} \leq\|f\|_{w_{p}^{s}} \leq\left((n+p) v_{n}\right)^{1 / p}\|f\|_{b_{p}^{s}},
$$

where $v_{n}$ is the volume of the unit ball in $\mathbb{R}^{n}$.

Proof. Define $\bar{\omega}_{p}(f, \delta)=\left(\delta^{-n} \int_{|h| \leq \delta} I_{p}^{p}(h) d h\right)^{1 / p}$. It is easy to see that

$$
v_{n}^{1 / p} 2^{-n-2} \omega_{p}(f, \delta) \leq \bar{\omega}_{p}(f, \delta) \leq v_{n}^{1 / p} \omega_{p}(f, \delta) \quad(\delta>0) .
$$


Next, by Fubini's theorem,

$$
\|f\|_{w_{p}^{s}}^{p}=(n+s p) \int_{0}^{\infty}\left(t^{-s} \bar{\omega}_{p}(f, t)\right)^{p} \frac{d t}{t} .
$$

Thus, applying (10), we obtain the desired result.

The following proposition is also based on simple and well known arguments.

Proposition 2.4. For any $f \in W_{p}^{1}\left(\mathbb{R}^{n}\right)$,

$$
\lim _{\delta \rightarrow+0} \frac{\omega_{p}(f, \delta)}{\delta}=\|\nabla f\|_{p} \quad(1 \leq p<\infty) .
$$

Proof. For each $h \in \mathbb{R}^{n}$ and almost every $x \in \mathbb{R}^{n}$,

$$
f(x+h)-f(x)=\int_{0}^{1} \nabla f(x+t h) \cdot h d t
$$

(see [9, p. 135]). By Minkowski's inequality we get $I_{p}(h) \leq|h|\|\nabla f\|_{p}$, and hence

$$
\omega_{p}(f, \delta) \leq \delta\|\nabla f\|_{p} \quad(\delta>0) .
$$

On the other hand, for any $\varepsilon>0$ there exists an infinitely differentiable function $f_{\varepsilon}$ with a compact support such that

$$
\left\|\nabla\left(f-f_{\varepsilon}\right)\right\|_{p}<\varepsilon .
$$

It is easy to see that

$$
\mu_{\varepsilon}(\delta)=\sup _{|h|=\delta}\left\|f_{\varepsilon}(\cdot+h)-f_{\varepsilon}(\cdot)-\nabla f_{\varepsilon}(\cdot) \cdot h\right\|_{p} / \delta \rightarrow 0 \quad \text { as } \delta \rightarrow 0 .
$$

Thus, there exists $\delta_{\varepsilon}>0$ such that $\mu_{\varepsilon}(\delta)<\varepsilon$ for all $0<\delta<\delta_{\varepsilon}$. Further, by (12) and (13),

$$
\omega_{p}\left(f_{\varepsilon}, \delta\right) \leq \omega_{p}(f, \delta)+\varepsilon \delta .
$$

Therefore, for all $0<\delta<\delta_{\varepsilon}$ we get

$$
\|\nabla f\|_{p} \leq\left\|\nabla f_{\varepsilon}\right\|_{p}+\varepsilon \leq \mu_{\varepsilon}(\delta)+\omega_{p}\left(f_{\varepsilon}, \delta\right) / \delta+\varepsilon \leq \omega_{p}(f, \delta) / \delta+3 \varepsilon .
$$

Together with (12), this yields (11).

The last proposition easily implies that the limiting relation (3) still holds for our choice of the definition of the Besov space. Moreover, we get a more general result.

Proposition 2.5. For any $f \in W_{p}^{1}\left(\mathbb{R}^{n}\right)$,

$$
\lim _{s \rightarrow 1-0}(1-s)^{1 / \theta}\|f\|_{b_{p, \theta}^{s}}=\left(\frac{1}{\theta}\right)^{1 / \theta}\|\nabla f\|_{L^{p}} \quad(1 \leq p, \theta<\infty) .
$$


Proof. Set $A=\|\nabla f\|_{L^{p}}$. Fix $\varepsilon>0$. By (11), there exists $\delta>0$ such that $\left|\left(\omega_{p}(f, t) / t\right)^{\theta}-A^{\theta}\right|<\varepsilon$ for any $t \in(0, \delta)$. Hence,

$$
\begin{aligned}
\left|(1-s)\|f\|_{b_{p, \theta}^{s}}^{\theta}-A^{\theta} / \theta\right| \leq & (1-s) \int_{0}^{\delta} t^{(1-s) \theta-1}\left|\left(\omega_{p}(f, t) / t\right)^{\theta}-A^{\theta}\right| d t \\
& +\frac{A^{\theta}}{\theta}\left|\delta^{(1-s) \theta}-1\right|+(1-s) \int_{\delta}^{\infty}\left(t^{-s} \omega_{p}(f, t)\right)^{\theta} \frac{d t}{t} \\
\leq & \frac{\varepsilon}{\theta} \delta^{(1-s) \theta}+\frac{A^{\theta}}{\theta}\left|\delta^{(1-s) \theta}-1\right|+\frac{2\|f\|_{p}^{\theta}}{s \theta \delta^{s \theta}}(1-s) .
\end{aligned}
$$

Now we can choose $\sigma>0$ such that

$$
\frac{\varepsilon}{\theta} \delta^{(1-s) \theta}+\frac{A^{\theta}}{\theta}\left|\delta^{(1-s) \theta}-1\right|+\frac{2\|f\|_{p}^{\theta}}{s \theta \delta^{s \theta}}(1-s)<3 \varepsilon
$$

for any $s \in(1-\sigma, 1)$, which proves (14).

Finally, the proof of our main result is based on the following theorem [6].

Theorem 2.6. Let $1 \leq p<q<\infty$ and $\gamma \equiv n(1 / p-1 / q)<1$. If either $p>1, n \geq 1$ or $p \geq 1, n \geq 2$, then for any $\delta>0$,

$$
\left(\int_{\delta}^{\infty}\left(t^{\gamma-1} \omega_{q}(f, t)\right)^{p} \frac{d t}{t}\right)^{1 / p} \leq c_{q, p, n} \delta^{\gamma-1}\left(\int_{0}^{\delta}\left(t^{-\gamma} \omega_{p}(f, t)\right)^{q} \frac{d t}{t}\right)^{1 / q}
$$

2.3. Rearrangements. The non-increasing rearrangement of a measurable function $f$ on $\mathbb{R}^{n}$ is defined by

$$
f^{*}(t)=\sup _{|E|=t} \inf _{x \in E}|f(x)| \quad(0<t<\infty)
$$

Define also

$$
f^{* *}(t)=\frac{1}{t} \int_{0}^{t} f^{*}(u) d u \quad(t>0)
$$

Let $0<p, r<\infty$. A measurable function $f$ belongs to the Lorentz space $L^{p, r}\left(\mathbb{R}^{n}\right)$ if

$$
\|f\|_{p, r} \equiv\left(\int_{0}^{\infty}\left(t^{1 / p} f^{*}(t)\right)^{r} \frac{d t}{t}\right)^{1 / r}<\infty .
$$

It is well known (see, e.g., [13, p. 192]) that

$$
\|f\|_{p, \nu} \leq\left(\frac{p}{\nu}\right)^{1 / \nu}\left(\frac{r}{p}\right)^{1 / r}\|f\|_{p, r} \quad(0<p<\infty, r<\nu) .
$$

The following theorem was proved in [5]. It plays a key role in our proof of (6). 
Theorem 2.7. Suppose that $f \in L^{p}\left(\mathbb{R}^{n}\right), 1 \leq p<\infty$. Then for any $\tau, \delta>0$,

and

$$
\int_{0}^{\tau}\left(f^{*}(u)-f^{*}(\tau)\right)^{p} d u \leq 2 \omega_{p}^{p}\left(f, \tau^{1 / n}\right)
$$

$$
\int_{\delta^{n}}^{\infty} t^{-p / n} \int_{0}^{t}\left(f^{*}(u)-f^{*}(t)\right)^{p} d u \frac{d t}{t} \leq c_{p, n} \omega_{p}^{p}(f, \delta) \delta^{-p} .
$$

Using (17) and Hölder's inequality, we immediately get the estimate

$$
f^{* *}(t)-f^{*}(t) \leq 2^{1 / p} t^{-1 / p} \omega_{p}\left(f, t^{1 / n}\right) .
$$

Note that in the one-dimensional case this was first proved by Ul'yanov [14].

We will also use the following result mentioned in the Introduction.

Theorem 2.8. Let $0<s<1$ and $1 \leq p<n / s$. Suppose also that $1 \leq \theta<\infty$. Then for any $f \in B_{p, \theta}^{s}\left(\mathbb{R}^{n}\right)$ we have

$$
\|f\|_{q^{*}, \theta} \leq c\|f\|_{b_{p, \theta}^{s}} \quad\left(q^{*}=\frac{n p}{n-s p}\right)
$$

where $c=2^{1 / p} n^{1 / \theta} q^{*}$.

This theorem was proved in $[4,12]$. In order to get the explicit value of the constant $c$ we will give an independent proof. Namely, applying (19), we obtain

$$
f^{* *}(t)=\int_{t}^{\infty}\left[f^{* *}(u)-f^{*}(u)\right] \frac{d u}{u} \leq 2^{1 / p} \int_{t}^{\infty} u^{-1-1 / p} \omega_{p}\left(f, u^{1 / n}\right) d u .
$$

From this and (8) we get

$$
\begin{aligned}
\|f\|_{q^{*}, \theta}^{\theta} & \leq 2^{\theta / p} \int_{0}^{\infty} t^{\theta / q^{*}-1}\left(\int_{t}^{\infty} u^{-1-1 / p} \omega_{p}\left(f, u^{1 / n}\right) d u\right)^{\theta} d t \\
& \leq\left(2^{1 / p} q^{*}\right)^{\theta} \int_{0}^{\infty} t^{\theta\left(1 / q^{*}-1 / p\right)} \omega_{p}^{\theta}\left(f, t^{1 / n}\right) \frac{d t}{t} \\
& =\left(2^{1 / p} q^{*}\right)^{\theta} n \int_{0}^{\infty}\left(t^{-s} \omega_{p}(f, t)\right)^{\theta} \frac{d t}{t}
\end{aligned}
$$

which yields (20).

\section{Main result}

Proof of Theorem 1.1. Set $\omega_{p}(f, t)=\omega(t), \omega_{q}(f, t)=\eta(t)$, and

$$
I \equiv\|f\|_{b_{q, \theta}^{s-\gamma}}^{\theta}=\int_{0}^{\infty}\left(t^{-(s-\gamma)} \omega_{q}(f, t)\right)^{\theta} \frac{d t}{t} .
$$


First we suppose that $\theta \leq p$. Using Fubini's theorem, we have

$$
I=p(1-s) \int_{0}^{\infty}\left(\int_{u}^{\infty}\left(t^{\gamma-1-1 / p} \eta(t)\right)^{\theta} t^{-\alpha} d t\right) u^{p(1-s)} \frac{d u}{u},
$$

where $\alpha=(1-\theta / p)(1+p(1-s))$. Next, applying (in the case $\theta<p)$ Hölder's inequality, we get

$$
\begin{aligned}
\int_{u}^{\infty}\left(t^{\gamma-1-1 / p} \eta(t)\right)^{\theta} t^{-\alpha} d t & \leq\left(\int_{u}^{\infty}\left(t^{\gamma-1} \eta(t)\right)^{p} \frac{d t}{t}\right)^{\theta / p}\left(\int_{u}^{\infty} \frac{d t}{t^{1+p(1-s)}}\right)^{1-\theta / p} \\
& =[p(1-s)]^{\theta / p-1} u^{(\theta-p)(1-s)}\left(\int_{u}^{\infty}\left(t^{\gamma-1} \eta(t)\right)^{p} \frac{d t}{t}\right)^{\theta / p} .
\end{aligned}
$$

It follows that

$$
I \leq p^{\theta / p}(1-s)^{\theta / p} \int_{0}^{\infty} u^{\theta(1-s)}\left(\int_{u}^{\infty}\left(t^{\gamma-1} \eta(t)\right)^{p} \frac{d t}{t}\right)^{\theta / p} \frac{d u}{u} .
$$

Applying (15), we obtain

$$
\int_{0}^{\infty} u^{\theta(1-s)}\left(\int_{u}^{\infty}\left(t^{\gamma-1} \eta(t)\right)^{p} \frac{d t}{t}\right)^{\theta / p} \frac{d u}{u} \leq c^{\theta} \int_{0}^{\infty} u^{-\theta(s-\gamma)}\left(\int_{0}^{u}\left(t^{-\gamma} \omega(t)\right)^{q} \frac{d t}{t}\right)^{\theta / q} \frac{d u}{u},
$$

where $c$ is a constant depending only on $p, q$, and $n$. Now we use the fact that the function $\omega(t) / t$ is quasi-decreasing with constant 2 . Therefore, using (9) with $\beta=q(1-\gamma)-1$, we get

$$
\begin{aligned}
& \int_{0}^{\infty} u^{-\theta(s-\gamma)}\left(\int_{0}^{u}\left(t^{-\gamma} \omega(t)\right)^{q} \frac{d t}{t}\right)^{\theta / q} \frac{d u}{u} \\
& \quad \leq \frac{\left(2^{q} q(1-\gamma)\right)^{1-\theta / q}}{(s-\gamma) q} \int_{0}^{\infty}\left(u^{-s} \omega(u)\right)^{\theta} \frac{d u}{u}=\frac{\left(2^{q} q(1-\gamma)\right)^{1-\theta / q}}{(s-\gamma) q}\|f\|_{b_{p, \theta}^{s}}^{\theta} .
\end{aligned}
$$

From this and from (21) we have

$$
I \leq A \frac{(1-s)^{\theta / p}}{s-\gamma}\|f\|_{b_{p, \theta}^{\theta}}^{\theta}
$$

( $A$ depends only on $p, q, \theta$ and $n$ ).

Assume now that $\theta>p$. First, by Fubini's theorem,

$$
I=\theta(1-s) \int_{0}^{\infty}\left(\int_{u}^{\infty}\left(t^{\gamma-1} \eta(t)\right)^{\theta} \frac{d t}{t}\right) u^{\theta(1-s)} \frac{d u}{u} .
$$

Further, since $\eta$ is non-decreasing,

$$
t^{\gamma-1} \eta(t) \leq\left((1-\gamma) p \int_{t}^{\infty}\left(u^{\gamma-1} \eta(u)\right)^{p} \frac{d u}{u}\right)^{1 / p} \quad(t>0) .
$$


Applying this inequality, we get

$$
\begin{aligned}
\int_{u}^{\infty}\left(t^{\gamma-1} \eta(t)\right)^{\theta} \frac{d t}{t} & =\int_{u}^{\infty}\left(t^{\gamma-1} \eta(t)\right)^{\theta-p}\left(t^{\gamma-1} \eta(t)\right)^{p} \frac{d t}{t} \\
& \leq 2^{\theta-p}\left(\int_{u}^{\infty}\left(t^{\gamma-1} \eta(t)\right)^{p} \frac{d t}{t}\right)^{\theta / p} .
\end{aligned}
$$

Therefore, from this and (15),

$$
\begin{aligned}
I & \leq c(1-s) \int_{0}^{\infty} u^{\theta(1-s)}\left(\int_{u}^{\infty}\left(t^{\gamma-1} \eta(t)\right)^{p} \frac{d t}{t}\right)^{\theta / p} \frac{d u}{u} \\
& \leq c(1-s) \int_{0}^{\infty} u^{\theta(\gamma-s)}\left(\int_{0}^{u}\left(t^{-\gamma} \omega(t)\right)^{q} \frac{d t}{t}\right)^{\theta / q} \frac{d u}{u} .
\end{aligned}
$$

It remains to use (9) in the case $\theta<q$ or (7) if $\theta \geq q$, to obtain

$$
I \leq c \frac{1-s}{s-\gamma}\|f\|_{b_{p, \theta}^{s}}^{\theta}
$$

where $c$ depends only on $q, p, \theta$, and $n$.

REMARK 3.1. It is easy to show that the assertion of Theorem 1.1 fails in the case $p=n=1$. Indeed, assume that (2) holds for $p=n=1$. Let $f=\chi_{(0,1)}$. A simple computation shows that

$$
\omega_{p}\left(\chi_{(0,1)}, t\right)=2^{1 / p} \min \left(t^{1 / p}, 1\right) .
$$

Hence, $\left\|\chi_{(0,1)}\right\|_{b_{1, \theta}^{s}}=2\left(\frac{1}{\theta s(1-s)}\right)^{1 / \theta}$ and

$$
\left\|\chi_{(0,1)}\right\|_{b_{q, \theta}^{s-\gamma}}=2^{1 / q}\left(\frac{1-\gamma}{\theta(1-s)(s-\gamma)}\right)^{1 / \theta} .
$$

Let, for example, $q=2$ and $1 / 2<s<1$. Then we get $c \leq 1-s$ for some fixed $c>0$, which contradicts (2) as $s \rightarrow 1$.

Remark 3.2. The exponent $1 / \theta^{*}$ in (2) is sharp in a sense. First assume that $\theta<p$. Let $\alpha=2-s-n / p$. We may suppose that $\alpha \neq 0$. Set

$$
f(x)=\frac{1}{\alpha}\left(1-|x|^{\alpha}\right) \chi_{B_{0}}(x),
$$

where $B_{0}$ is the unit ball in $\mathbb{R}^{n}$. Then a calculation shows that $\|f\|_{p}^{p} \leq$ $c_{p, n}$, where the constant $c_{p, n}$ does not depend on $\alpha$. Further, $|\nabla f(x)|=$ $|x|^{\alpha-1} \chi_{B_{0}}(x)$ and $\|\nabla f\|_{p}=c_{p, n}(1-s)^{-1 / p}$. Thus, $\omega_{p}(f, t) \leq c_{p, n}(1-s)^{-1 / p} t$ for $0<t \leq 1$ and $\omega_{p}(f, t) \leq 2\|f\|_{p} \leq c_{p, n}$ for $t \geq 1$. Hence,

$$
\|f\|_{b_{p, \theta}^{s}} \leq c_{p, n}(1-s)^{-1 / p-1 / \theta} .
$$


On the other hand, if $0<t<1, t / 8 \leq|x| \leq t / 4$, and $|h|=t$, then $|f(x)-f(x+h)| \geq 2^{-5} t^{\alpha}$, and thus, $\omega_{q}(f, t) \geq c t^{\alpha+n / q} \quad(0<t<1)$, where $c$ is a positive constant that does not depend on $s$. From this and (22),

$$
\|f\|_{b_{q, \theta}^{s-\gamma}} \geq c(1-s)^{-1 / \theta} \geq c^{\prime}(1-s)^{-1 / p}\|f\|_{b_{p, \theta}^{s}}
$$

$\left(c^{\prime}\right.$ is positive and does not depend on $s$ ). This shows that the exponent $1 / p$ of $1-s$ in $(2)$ is sharp $(\theta<p)$.

If $\theta>p$, it suffices to consider the function $f(x)=\max (1-|x|, 0)$.

REMARK 3.3. If either $p \neq 1$ or $n \neq 1,1 / 2<s<1$, and $1 \leq p<n / s$, then Theorem 1.1 easily implies (6):

$$
\|f\|_{q^{*}, p}^{p} \leq c_{p, n} \frac{1-s}{(n-s p)^{p}}\|f\|_{b_{p}^{s}}^{p} \quad\left(q^{*}=\frac{n p}{n-s p}\right),
$$

which is a more precise result than (4). Indeed, set $\theta=p, \gamma=s-1 / 2$, and $q=n p /(n-\gamma p)$. Then $q^{*}=n q /(n-q / 2)$. Applying Theorems 2.8 and 1.1, we have

$$
\|f\|_{q^{*}, p}^{p}=\|f\|_{n q /(n-q / 2), p}^{p} \leq \frac{c_{n, p}}{(n-s p)^{p}}\|f\|_{b_{q, p}^{1 / 2}} \leq c_{p, n} \frac{1-s}{(n-s p)^{p}}\|f\|_{b_{p}^{s}}^{p},
$$

as required.

In the next section we will show a different way of proving (6) for any $p$ and $n$.

4. On the Bourgain-Brezis-Mironescu inequality. In Section 2 we applied inequality (19) to prove Theorem 2.8 . We now use sharper rearrangement estimates (Theorem 2.7) to prove the following.

TheOrem 4.1. Let $0<s<1$ and $1 \leq p<n / s$. Then for any $f \in$ $B_{p}^{s}\left(\mathbb{R}^{n}\right)$,

$$
\|f\|_{L^{q^{*}, p\left(\mathbb{R}^{n}\right)}}^{p} \leq c_{p, n} \frac{s(1-s)}{(n-s p)^{p}}\|f\|_{b_{p}^{s}\left(\mathbb{R}^{n}\right)}^{p} \quad\left(q^{*}=\frac{n p}{n-s p}\right) .
$$

Observe that, by (16),

$$
\|f\|_{L^{q^{*}}}^{p} \leq \frac{n-s p}{n}\|f\|_{L^{q^{*}, p}}^{p}
$$

and hence (23) immediately implies (4) with the constant

$$
c_{p, n} s(1-s)(n-s p)^{1-p} \text {. }
$$

Proof of Theorem 4.1. Suppose first that $1 / 2 \leq s<1$. Using the identity

$$
f^{* *}(t)=\int_{t}^{\infty}\left(f^{* *}(\tau)-f^{*}(\tau)\right) \frac{d \tau}{\tau},
$$


we obtain

$$
\begin{aligned}
\|f\|_{q^{*}, p}^{p} & =\int_{0}^{\infty}\left(f^{*}(t)\right)^{p} \frac{d t}{t^{s p / n}} \leq \int_{0}^{\infty}\left(f^{* *}(t)\right)^{p} \frac{d t}{t^{s p / n}} \\
& \leq\left(\frac{n p}{n-s p}\right)^{p} \int_{0}^{\infty}\left(f^{* *}(t)-f^{*}(t)\right)^{p} \frac{d t}{t^{s p / n}} \\
& =\left(\frac{n p}{n-s p}\right)^{p}(1-s) p \int_{0}^{\infty} u^{(1-s) p} \int_{u^{n}}^{\infty} t^{-p / n}\left(f^{* *}(t)-f^{*}(t)\right)^{p} d t \frac{d u}{u} \\
& \leq\left(\frac{n p}{n-s p}\right)^{p}(1-s) p \int_{0}^{\infty} u^{(1-s) p} \int_{u^{n}}^{\infty} t^{-p / n} \int_{0}^{t}\left(f^{*}(\xi)-f^{*}(t)\right)^{p} d \xi \frac{d t}{t} \frac{d u}{u} \\
& \leq c_{p, n} \frac{1-s}{(n-s p)^{p}} \int_{0}^{\infty}\left(u^{-s} \omega_{p}(f, u)\right)^{p} \frac{d u}{u}=c_{p, n} \frac{1-s}{(n-s p)^{p}}\|f\|_{b_{p}^{s}}^{p}
\end{aligned}
$$

(we applied Hardy inequality (8), Fubini's theorem, Hölder's inequality and (18), respectively).

Consider now the case $0<s<1 / 2$. Using the elementary estimate

$$
f^{*}(t) \leq \int_{t}^{\infty}\left(f^{*}(\tau / e)-f^{*}(\tau)\right) \frac{d \tau}{\tau},
$$

we have

$$
\begin{aligned}
\|f\|_{q^{*}, p}^{p} & \leq\left(\frac{n p}{n-s p}\right)^{p} \int_{0}^{\infty}\left(f^{*}(t / e)-f^{*}(t)\right)^{p} \frac{d t}{t^{s p / n}} \\
& =\left(\frac{n p}{n-s p}\right)^{p} \frac{s p}{n} \int_{0}^{\infty} u^{-s p / n} \int_{0}^{u}\left(f^{*}(t / e)-f^{*}(t)\right)^{p} d t \frac{d u}{u} \\
& \leq\left(\frac{n p}{n-s p}\right)^{p} \frac{s p}{n} \int_{0}^{\infty} u^{-s p / n} \int_{0}^{u}\left(f^{*}(t / e)-f^{*}(u)\right)^{p} d t \frac{d u}{u} \\
& \leq e\left(\frac{n p}{n-s p}\right)^{p} \frac{s p}{n} \int_{0}^{\infty} u^{-s p / n} \int_{0}^{u}\left(f^{*}(t)-f^{*}(u)\right)^{p} d t \frac{d u}{u} \\
& \leq 2 e\left(\frac{n p}{n-s p}\right)^{p} \frac{s p}{n} \int_{0}^{\infty} u^{-s p / n} \omega_{p}^{p}\left(f, u^{1 / n}\right) \frac{d u}{u} \\
& =2 e n^{p} p^{p+1} \frac{s}{(n-s p)^{p}}\|f\|_{b_{p}^{s}}^{p}
\end{aligned}
$$

(here we used Hardy inequality (8), Fubini's theorem and (17)). Combining both cases, we get (23). 
Remark 4.2. The cases $0<s<1 / 2$ and $1 / 2 \leq s<1$ correspond to "small" and "big" smoothness, respectively. Inequality (17) is sharp only in the case of "small" smoothness, while (18) is sharp in both cases (see [5] for details). Therefore, to prove Theorem 4.1 for small $s$, it suffices to apply (17), while the case of big $s$ requires a stronger inequality (18).

REMARK 4.3. Observe that in the case $p=n=1$ the proof of (23) is extremely simple. Indeed, exactly as in [5, Theorem 1], one can prove that

$$
f^{* *}(t) \leq 2 \omega_{1}(f, t) / t
$$

Therefore,

$$
\|f\|_{1 /(1-s), 1}=s \int_{0}^{\infty} f^{* *}(t) \frac{d t}{t^{1 / s}} \leq 2 s \int_{0}^{\infty} \omega_{1}(f, t) \frac{d t}{t^{1+1 / s}}=2 s\|f\|_{b_{1}^{s}},
$$

and (23) is proved. We also outline, for the sake of completeness, the proof of (24). It suffices to assume that $f$ has a compact support. Then for any $x \in \mathbb{R}$ and all $t>0$,

$$
\begin{aligned}
|f(x)| \leq & \left|f(x)-\frac{1}{t} \int_{0}^{t} f(x+y) d y\right| \\
& +\frac{1}{t} \sum_{j=0}^{\infty}\left|\int_{j t}^{(j+1) t} f(x+y) d y-\int_{(j+1) t}^{(j+2) t} f(x+y) d y\right| \\
\leq & \frac{1}{t} \int_{0}^{t}|f(x+y)-f(x)| d y+\frac{1}{t} \int_{\mathbb{R}}|f(y+t)-f(y)| d y .
\end{aligned}
$$

Hence,

$$
f^{* *}(t) \leq \frac{1}{t^{2}} \int_{0}^{t}\|f(y+\cdot)-f(\cdot)\|_{1} d y+\frac{1}{t} \int_{\mathbb{R}}|f(y+t)-f(y)| d y \leq 2 \omega_{1}(f, t) / t,
$$

and $(24)$ is proved.

Acknowledgments. The first named author was partially supported by grant BFM 2000-0206-C04-03 of the DGI, Spain.

We are grateful to the referee for his/her useful remarks.

\section{References}

[1] J. Bourgain, H. Brezis and P. Mironescu, Another look at Sobolev spaces, in: Optimal Control and Partial Differential Equations. In honour of Professor Alain Bensoussan's 60th Birthday, J. L. Menaldi, E. Rofman and A. Sulem (eds.), IOS Press, Amsterdam, 2001, 439-455. 
[2] J. Bourgain, H. Brezis and P. Mironescu, Limiting embedding theorems for $W^{s, p}$ when $s \uparrow 1$ and applications, J. Analyse Math. 87 (2002), 77-101.

[3] O. V. Besov, V. P. Il'in and S. M. Nikol'skiǔ, Integral Representations of Functions and Embedding Theorems, Vols. 1-2, Winston, Washington, DC, Halsted Press, New York, 1978-1979.

[4] C. S. Herz, Lipschitz spaces and Bernstein's theorem on absolutely convergent Fourier transforms, J. Math. Mech. 18 (1968), 283-323.

[5] V. I. Kolyada, Estimates of rearrangements and embedding theorems, Mat. Sb. 136 (1988), 3-23 (in Russian); English transl.: Math. USSR-Sb. 64 (1989), 1-21.

[6] -, On relations between moduli of continuity in different metrics, Trudy Mat. Inst. Steklova 181 (1988), 117-136 (in Russian); English transl.: Proc. Steklov Inst. Math. 181 (1989), 127-148.

[7] - Rearrangement of functions and embedding of anisotropic spaces of Sobolev type, East J. Approx. 4 (1998), 111-199.

[8] L. D. Kudryavtsev and S. M. Nikol'skiǔ, Spaces of Differentiable Functions of Several Variables and Embedding Theorems, Encyclopedia Math. Sci. 26, Springer, Berlin, 1991, 4-140.

[9] E. H. Lieb and M. Loss, Analysis, Grad. Stud. in Math. 14, Amer. Math. Soc., 1997.

[10] V. Maz'ya and T. Shaposhnikova, On the Bourgain, Brezis, and Mironescu theorem concerning limiting embeddings of fractional Sobolev spaces, J. Funct. Anal. 195 (2002), 230-238.

[11] S. M. Nikol'skiı̌, Approximation of Functions of Several Variables and Embedding Theorems, Springer, Berlin, 1975.

[12] J. Peetre, Espaces d'interpolation et théorème de Soboleff, Ann. Inst. Fourier (Grenoble) 16 (1966), no. 1, 279-317.

[13] E. M. Stein and G. Weiss, Introduction to Fourier Analysis on Euclidean Spaces, Princeton Univ. Press, 1971.

[14] P. L. Ul'yanov, Embedding of certain function classes $H_{p}^{\omega}$, Izv. Akad. Nauk SSSR Ser. Mat. 32 (1968), 649-686; English transl.: Math. USSR-Izv. 2 (1968), 601-637.

[15] I. Wik, Symmetric rearrangement of functions and sets in $\mathbb{R}^{n}$, Report no. 1 (1977), Dept. of Math., Univ. of Umeå, 1-36.

Department of Mathematics

Karlstad University

65188 Karlstad, Sweden

E-mail: viktor.kolyada@kau.se
Department of Mathematics Bar-Ilan University 52900 Ramat Gan, Israel E-mail: aklerner@netvision.net.il

Received January 5, 2004

Revised version August 17, 2004 